Objectives: We compared clinical features of AAV with renal involvement with patients without renal involvement.

Methods: We conducted an observational study of 104 patients with AAV (12 eosinophilic granulomatosis with polyangiitis, 23 granulomatosis with polyangiitis (GPA), 66 microscopic polyangiitis, 3 renal limited vasculitis) between in 2008 to 2016 in Nagasaki University Hospital. Using medical records, we analyzed the patients' baseline variables, laboratory data, clinical symptoms, and therapeutic outcomes after treatments including episodes of relapses, initiations of dialysis, and death. Renal involvement was defined as the state with estimated glomerular filtration rate $<60 \mathrm{~mL} / \mathrm{min} / 1.73 \mathrm{~m}^{2}$ or microscopic hematuria (2+ or greater) which were not caused by renal diseases except for AAV.

Results: Sixty-nine patients had renal involvement. Patients with renal involvement group had higher median age at diagnosis than patients without renal involvement group ( 75 years vs. 66 years, $p<0.001$ ). Patients with renal involvement included fewer GPA patients compared to other AAV types. Patients with renal involvement had lower hemoglobin levels (10.3 g/dL vs. $12.3 \mathrm{~g} / \mathrm{dL})$ and lower platelet levels $\left(23.7 \times 10^{4} / \mu \mathrm{L}\right.$ vs. $\left.28.7 \times 10^{4} / \mu \mathrm{L}\right)$. Patients with renal involvement had higher erythrocyte sedimentation rate $(78 \mathrm{~mm} / \mathrm{h}$ vs. $20 \mathrm{~mm} / \mathrm{h})$, MPO-ANCA titers $(116 \mathrm{U} / \mathrm{mL}$ vs. $58 \mathrm{U} / \mathrm{mL})$ and urine protein levels $(0.81 \mathrm{~g} / \mathrm{gCr}$ vs. $0.15 \mathrm{~g} / \mathrm{gCr})$. Patients with renal involvement had lower $\mathrm{C} 3$ levels, but $\mathrm{CH} 50$ and $\mathrm{C} 4$ levels did not differ between in two groups. There were no differences in treatments including doses of prednisolone and use of methylprednisolone pulse and cyclophosphamide between in two groups. Multivariable regression analysis revealed that age at diagnosis is the most significant explanatory variable to renal involvement. Nineteen percent of patients with renal involvement had initiations of dialysis. Multivariable analysis demonstrated estimated glomerular filtration rate at diagnosis is the most significant explanatory variable to initiations of dialysis $(p=0.010)$. Receiver operating characteristic curve showed the cutoff level of estimated glomerular filtration rate to distinguish initiations of dialysis was $37 \mathrm{~mL} / \mathrm{min} / 1.73$ $\mathrm{m}^{2}$ (sensitivity $=79 \%$, specificity $=70 \%$, area under the curve $=0.80$ ). Assessed by a log-rank test, overall survival rate did not differ between in two groups $(p=0.29)$. Conclusions: Patients with renal involvement had higher age at diagnosis. Patients with renal involvement included fewer GPA patients. Patients with renal involvement had lower C3 levels. Estimated glomerular filtration rate at diagnosis is the most significant explanatory variable to initiations of dialysis.

Disclosure of Interest: None declared

DOI: 10.1136/annrheumdis-2017-eular.2859

\section{THU0304 VASCULITIS PATIENTS ADMITTED TO INTENSIVE CARE UNIT: IMPLICATIONS FROM A SINGLE-CENTER RETROSPECTIVE STUDY}

A. Watad ${ }^{1}$, O. Shovman ${ }^{2}$, N.L. Bragazzi ${ }^{3}$, Y. Shoenfeld ${ }^{4}$, H. Amital ${ }^{5}$, Y. Haviv ${ }^{6}$. ${ }^{1}$ Internal Medicine B; ${ }^{2}$ Department B, Sheba Medical Center, Tel Aviv, Israel; ${ }^{3}$ Department of Health Sciences, Genova university, Genoa, Italy; ${ }^{4}$ Zabludowicz center for Autoimmune Diseases; ${ }^{5}$ Sheba Medical Center, Tel Aviv, Israel; ${ }^{6}$ Department of General Intensive Care, Sheba Medical Center, Tel Aviv, Israel

Background: Vasculitides are a heterogeneous group of disorders that are characterized by the inflammation of the vasculature vessels. Vasculitides may present as a life-threatening condition and cause higher rates of morbidity and mortality. There are few studies assessing the outcome and prognosis of patients with vasculitides admitted to the intensive care (ICU).

Objectives: To assess the outcome of vasculitides patients required admission to ICU and to identify factors associated with mortality

Methods: A retrospective study was carried out, including all patients who were diagnosed with vasculitides and admitted to the ICU of the Sheba Medical Center, Tel-Hashomer, throughout the years 2000-2014. Continuous variables were computed as mean \pm standard deviation, whilst categorical variables were recorded as percentages, where appropriate. Student's t-test and chi-squared analyses were performed for investigating the impact of the clinical variables on mortality. Results: Twenty-five vasculitides patients admitted to the ICU were included in the present study (mean age $52 \pm 14 y$, sex ratio M/F: $12 / 13$ ). The mortality rate among these patients was $48 \%$. Leading causes for ICU admission were: infection (64\%), vasculitides exacerbation (34\%), and hemorrhage (16\%). Variables significantly associated with mortality were: the use of Rituximab prior to admission ICU $(p=0.039)$, involvement of the hemodynamic system $(p=0.024)$, the SOFA score $(p=0.041)$, blood infections during the first week at ICU $(p=0.018)$ and persisting after the first week $(p=0.007)$

Figure 1. Receiver Operating Characteristic (ROC) analysis of the predictive power of the Sequential Organ Failure Assessment (SOFA) score, in predicting the mortality of vasculitis patients admitted at intensity care unit (ICU), in terms of sensitivity and specificity. SOFA

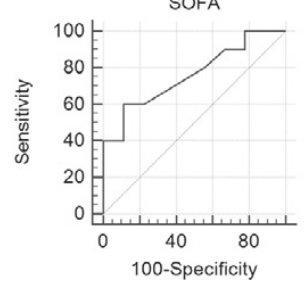

Conclusions: Our study confirms the high mortality rate among vasculitides patients and mainly among those requiring admission to ICU. SOFA score and preadmission treatment with rituximab have been found to be predictive of mortality. Disclosure of Interest: None declared

DOI: 10.1136/annrheumdis-2017-eular.4431

\section{THU0305 EPIDEMIOLOGY OF ANCA-ASSOCIATED VASCULITIS IN NORTHERN NORWAY}

A.T. Nilsen ${ }^{1}$, R.A. Watts ${ }^{2}$, W. Koldingsnes ${ }^{1} .{ }^{1}$ Department of Rheumatology, University Hospital of North Norway, Tromsø, Norway; ${ }^{2}$ Norwich Medical School, University of East Anglia, Norwich, United Kingdom

Background: The ANCA-associated vasculitides (AAV) have increased in prevalence since the 1980s, with granulomatosis with polyangiitis (GPA) being most prevalent in Caucasian population in circumpolar areas. This was also shown in a study on GPA in northern Norway between 1984 and 1998, which further showed an increasing incidence [1].

Objectives: The present study aimed to investigate the subsequent 15-year period in the same region, now including all the AAVs.

Methods: The study area has 11 hospitals, no private specialist in rheumatology or nephrology, and an adult population of 371928 . We retrospectively searched all hospital databases, using ICD-10 codes potentially compatible with AAV. Patients diagnosed with AAV from 1999 through 2013 according to the European Medicines Agency (EMEA) algorithm, and for GPA also the subgroup fulfilling the American College of Rheumatology (ACR) 1990 criteria, were included. For prevalence data, patients residing in the area, but with AAV diagnosis prior to 1999, were included too.

Results: Using the EMEA algorithm, 90 incident cases were classified as GPA 39 as microscopic polyangiitis (MPA) and 14 as eosinophilic granulomatosis with polyangiitis (EGPA). Within the GPA group, 78 patients also met the ACR criteria. The results for incidence and prevalence are given in Table 1:

\begin{tabular}{|c|c|c|c|c|c|c|c|c|}
\hline & & \multicolumn{4}{|c|}{$\begin{array}{c}\text { Annual } \\
\text { incidence/million }\end{array}$} & \multicolumn{3}{|c|}{$\begin{array}{c}\text { Point prevalence } \\
\text { at 31. Dec }\end{array}$} \\
\hline & & 1999-2003 & 2004-2008 & 2009-2013 & Total period & 2003 & 2008 & 2013 \\
\hline ACR & GPA & 11,4 & 16,7 & 12,5 & 13,6 & 149 & 210 & 235 \\
\hline EMEA & GPA & 11,4 & 19,9 & 15,7 & 15,8 & 149 & 226 & 261 \\
\hline EMEA & MPA & 2,7 & 6,5 & 11,0 & 6,8 & 10,8 & 31,9 & 63,3 \\
\hline EMEA & EGPA & 2,7 & 2,7 & 1,6 & 2,3 & 13,5 & 18,6 & 30,4 \\
\hline EMEA & All AAV & 16,8 & 29,0 & 28,2 & 24,9 & 173 & 276 & 354 \\
\hline
\end{tabular}

GPA (ACR) point prevalence per million

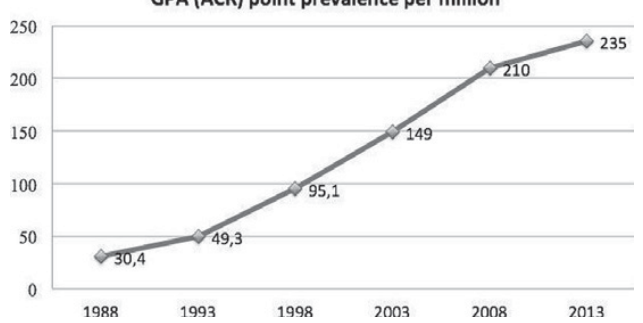

Conclusions: The GPA incidence and prevalence in this study are the highest reported. Though the incidence has stabilized, prevalence is still increasing, albeit at a decelerating rate (Graph 1). Moreover, the total AAV prevalence doubled in the last 10 years, exceeding previous estimates. Incidence of MPA and EGPA are both within the range found elsewhere. But the MPA incidence appears to be rising reminiscent of GPA before the turn of the century.

References:

[1] Koldingsnes W, Nossent $\mathrm{H}$. Epidemiology of Wegener's granulomatosis in northern Norway. Arthritis Rheum. 2000;43(11):2481-2487.

Disclosure of Interest: None declared

DOI: 10.1136/annrheumdis-2017-eular.4158

\section{THU0306 PREVALENCE AND CHARACTERISTICS OF NEUROPATHY IN PATIENTS WITH ANCA ASSOCIATED VASCULITIDES: DATA FROM THE DCVAS STUDY}

A. Bischof ${ }^{1,2,3}$, V.K. Jaeger ${ }^{4}$, M.P. Collins ${ }^{5}$, R.D.M. Hadden ${ }^{6}$, R. Luqmani ${ }^{7}$, R. Suppiah ${ }^{8}$, A. Craven ${ }^{7}$, T. Daikeler ${ }^{9} .{ }^{1}$ Neurology, University of California San Francisco, San Francisco, United States; ${ }^{2}$ Neurology, University Hospital Basel, Switzerland; ${ }^{3}$ Immunology Clinic; ${ }^{4}$ Rheumatology, University Hospital Basel, Basel, Switzerland; ${ }^{5}$ Neurology, Medical College of Wisconsin, Milwaukee, United States; ${ }^{6}$ Neurology, King's College Hospital, London; ${ }^{7}$ Nuffield Department of Orthopaedics, Rheumatology and Musculoskeletal Sciences, University of Oxford, Oxford, United Kingdom; ${ }^{8}$ Rheumatology, Auckland District Health Board, Auckland, New Zealand; ${ }^{9}$ Rheumatology, University Hospital Basel, Switzerland, Basel, Switzerland

Background: Epidemiological data on vasculitic neuropathy (VN) in ANCA associated vasculitides (AAV) are scarce and controversial. The Diagnostic and 